Revista Brasileira de Agricultura Irrigada v.5, $\mathrm{n}^{\circ} .1$, p.54-62, 2011

ISSN 1982-7679 (On-line)

Fortaleza, CE, INOVAGRI - http://www.inovagri.org.br/rbai

Protocolo 04409 - 28/10/2010 Aprovado em 03/03/2011

\title{
TANQUE EVAPORIMÉTRICO ALTERNATIVO E EQUAÇÕES PARA ESTIMATIVA DA EVAPOTRANSPIRAÇÃO DE REFERÊNCIA NA REGIÃO NORTE DE MG
}

\author{
Thiago Mendes Lisboa ${ }^{1,3}$, Carlos Henrique Batista ${ }^{1,3}$, Leonardo Angelo de Aquino ${ }^{2}$, \\ Heider Rodrigo Ferreira de Silva ${ }^{3}$, Vinícius Lopes de Melo ${ }^{1,4}$ e Valdeir Celestino Santos Junior ${ }^{3}$ \\ ${ }^{1}$ Tecnólogo em Irrigação e Drenagem, Instituto Federal do Norte de Minas Gerais (IFNMG) - Campus Januária, \\ Januária, MG. E-mails: thiagojanuaria@yahoo.com.br \\ ${ }^{1}$ Engenheiro Agrônomo, D. Sc. Fitotecnia, Professor, Universidade Federal de Viçosa (UFV) - Campus Rio \\ Paranaíba. BR 354, Km 310 (a 1300 m). E-mail: leonardo.aquino@ufv.br \\ ${ }^{3}$ Acadêmico de Agronomia, IFNMG - Campus Januária. Fazenda São Geraldo, s/n, Bom Jardim, CEP 39480- \\ 000, Januária, MG. E-mails: heiderrfs@yahoo.com.br; scvaldeir@ hotmail.com \\ ${ }^{4}$ Acadêmico de Engenharia Agrícola e Ambiental, IFNMG - Campus Januária. Fazenda São Geraldo, s/n, Bom \\ Jardim, CEP 39480-000, Januária, MG. E-mail: viniciuslopesmelo@ yahoo.com.br
}

\section{RESUMO}

A quantificação da evapotranspiração na região semiárida é de grande importância, visto que essa região apresenta grande ocorrência de períodos de déficit hídrico que limitam a produção agrícola. Objetivou-se com este trabalho avaliar a correlação entre a evapotranspiração de referência $\left(\mathrm{ET}_{0}\right)$, determinada pelo método de Penman-Monteith $\left(\mathrm{ET}_{0}-\mathrm{PM}\right)$, com a estimada pelos métodos do Tanque Classe A (ET $\left.{ }_{0}-\mathrm{TA}\right)$, de Linacre $\left(\mathrm{ET}_{0}-\mathrm{L}\right)$, de Hargreaves-Samani $\left(\mathrm{ET}_{0}-\mathrm{H} \& \mathrm{~S}\right)$, e a evaporação de um tanque alternativo denominado de Minitanque (EV - MT), na região Norte de Minas Gerais. Os dados foram coletados durante 12 meses de uma estação automática e de um minitanque alocados na Unidade de Pesquisa do Instituto Federal do Norte Minas Gerais, Campus Januária, sob coordenadas geográficas de latitude $15^{\circ} 28^{\prime} 55^{\prime}$ ' S e longitude $44^{\circ} 22^{\prime} 41^{\prime}$ ' W, altitude $474 \mathrm{~m}$. Foram ajustadas equações de regressão linear, tendo como variável independente, a $\mathrm{ET}_{0}-$ $\mathrm{PM}$, e, como variáveis dependentes, a $\mathrm{ET}_{0}-\mathrm{H} \& \mathrm{~S}$, a $\mathrm{ET}_{0}-\mathrm{L}$, a $\mathrm{ET}_{0}-\mathrm{TA}$ e a $\mathrm{EV}-\mathrm{MT}$. Os valores de $\mathrm{ET}_{0}-\mathrm{H} \& \mathrm{~S}, \mathrm{ET}_{0}-\mathrm{L}, \mathrm{ET}_{0}-\mathrm{TA}$ e $\mathrm{EV}$ - MT apresentaram boa correlação e superestimaram a $\mathrm{ET}_{0}-\mathrm{PM}$. As regressões entre a $\mathrm{ET}_{0}$ - $\mathrm{PM}$ (método padrão) e a $\mathrm{ET}_{0}$, calculadas pelos métodos estudados e, entre $\mathrm{ET}_{0}-\mathrm{PM}$ e a evaporação do minitanque, apresentaram maiores coeficientes de determinação no período sem chuvas.

Palavras-chave: Manejo de irrigação, Agrometeorologia, Demanda hídrica das culturas.

\section{ALTERNATIVE EVAPORIMETRIC TANK AND EQUATIONS FOR ESTIMATE OF REFERENCE EVAPOTRANSPIRATION IN THE NORTH OF MG}

\begin{abstract}
The evapotranspiration quantification in semiarid region of Brazil has great importance, since this region has a high occurrence of drought periods that limit agricultural production. The objective of this study is to evaluate the correlation between the reference evapotranspiration $\left(\mathrm{ET}_{0}\right)$, calculated by Penman-Monteith $\left(\mathrm{ET}_{0}-\mathrm{PM}\right)$, and the one estimated by the Class A tank $\left(\mathrm{ET}_{0}\right.$ -
\end{abstract}




\section{TANQUE EVAPORIMÉTRICO ALTERNATIVO E EQUAÇÕES PARA ESTIMATIVA DA EVAPOTRANSPIRAÇÃO DE REFERÊNCIA NA REGIÃO NORTE DE MG}

TA), of Linacre (ET $E_{0}-\mathrm{L}$ ), of Hargreaves-Samani $\left(\mathrm{ET}_{0}-\mathrm{H} \& \mathrm{~S}\right)$, and evaporation of an alternative tank called Mini tank (EV - MT) in the north of Minas Gerais, Brazil. Data were collected during 12 months from an automatic station and a mini tank allocated in the Research Unit of the Federal Institute of Northern of Minas Gerais, Campus Januária, under the geographical coordinates of latitude $15^{\circ} 28^{\prime} 55^{\prime \prime} \mathrm{S}$ and longitude $44^{\circ} 22^{\prime} 41^{\prime \prime} \mathrm{W}$, altitude $474 \mathrm{~m}$. Linear regression equations were fitted with $\mathrm{ET}_{0}-\mathrm{PM}$, independent variable, and as dependent variables, $\mathrm{ET}_{0}-\mathrm{H} \& \mathrm{~S}, \mathrm{ET}_{0}-\mathrm{L}$, ET0 - TA and EV - MT. The values of $\mathrm{ET}_{0}-\mathrm{H} \& \mathrm{~S}, \mathrm{ET}_{0}-\mathrm{L}, \mathrm{ET}_{0}$ - TA and EV - MT showed good correlation and overestimated to $\mathrm{ET}_{0}-\mathrm{PM}$. The regressions between $\mathrm{ET}_{0}-\mathrm{PM}$ (standard method) and $\mathrm{ET}_{0}$, calculated by the studied methods, and between $\mathrm{ET}_{0}-\mathrm{PM}$ and the mini tank evaporation showed higher coefficients of determination for the period without rain.

Keywords: Irrigation Management, Agrometeorology, and Crop Water Demand.

\section{INTRODUÇÃO}

A quantificação da evapotranspiração de referência $\left(\mathrm{ET}_{0}\right)$ é utilizada para determinar a demanda hídrica das culturas. Essa determinação é fundamental na elaboração de projetos e manejo de sistemas de irrigação. $\mathrm{Na}$ região semiárida, a quantificação da evapotranspiração é de grande importância visto que essa região apresenta grande ocorrência de períodos de déficit hídrico que limitam a produção agrícola (Henrique e Dantas, 2007).

$\mathrm{O}$ método padrão para estimar a evapotranspiração de referência é o de Penmam-Monteih, proposto no paper FAO 56 (Allen et al., 2006). Este método foi escolhido padrão porque ele se aproxima da $\mathrm{ET}_{0}$ estimada pelos métodos diretos. $\mathrm{O}$ método possui base física sólida e incorpora explicitamente parâmetros fisiológicos e aerodinâmicos.

O método de Penman-Monteith apresenta o inconveniente do grande número de elementos meteorológicos necessários. Assim, foram desenvolvidos métodos alternativos simplificados, de baixo custo, para determinar a $\mathrm{ET}_{0}$. Tais métodos apresentam boa correlação com o método padrão da FAO. Os métodos simplificados permitem aos agricultores que não têm acesso a todos os elementos climáticos determinar a $\mathrm{ET}_{0}$ a ser utilizada em projetos ou no manejo de irrigação (Silva et al., 2005; Henrique e Dantas, 2007; Oliveira et al., 2010).

Equações empíricas foram desenvolvidas para determinar a evapotranspiração de referência com base em dados meteorológicos. Entretanto, alguns dos métodos são aplicáveis apenas em determinadas condições climáticas e agronômicas e não podem ser aplicados em condições diferentes das que foram originalmente desenvolvidos (Allen et al., 2006).

Podem existir diferenças significativas entre a $\mathrm{ET}_{0}$ estimada por diferentes métodos, para uma mesma região. $\mathrm{A} \mathrm{ET}_{0}$ estimada pelo método de Linacre subestimou a dos métodos de Hargreaves, Penman-Monteith e o proposto por Henrique e Dantas (2007) em todas as estações do ano para Campina Grande - PB. O método proposto por Henrique e Dantas (2007) apresentou valores diários de evapotranspiração mais próximos aos estimados pelo modelo de Hargreaves em todas as estações do ano.

$\mathrm{O}$ tanque classe A tem sido muito empregado em manejo de irrigação devido à sua facilidade de operação e resultados satisfatórios para a estimativa da demanda hídrica das culturas (Bernardo et al., 2006; Oliveira et al., 2010). A evaporação da superfície livre da água fornece a evaporação do tanque. Pelo seu produto com coeficientes empíricos se obtém a $\mathrm{ET}_{0}$ (Allen et al., 2006).

$\mathrm{O}$ uso de tanques alternativos para medir a evaporação pode ser uma ferramenta útil para o manejo da irrigação, especialmente para pequenos agricultores que não têm acesso aos elementos meteorológicos necessários para o uso das equações. Estes mesmos tanques apresentam a vantagem da medição direta da evaporação, que se correlaciona com a evapotranspiração das culturas. 


\section{TANQUE EVAPORIMÉTRICO ALTERNATIVO E EQUAÇÕES PARA ESTIMATIVA DA EVAPOTRANSPIRAÇÃO DE REFERÊNCIA NA REGIÃO NORTE DE MG}

Objetivou-se com este trabalho comparar a $\mathrm{ET}_{0}$ determinada pelo método de PenmanMonteith com a estimada pelos métodos do Tanque Classe A, de Linacre, de Hargreaves-Samani e a evaporação um tanque alternativo denominado de Minitanque em um período de 12 meses, na região Norte de Minas Gerais.

\section{MATERIAL E MÉTODOS}

O trabalho foi conduzido no período de 1 de novembro de 2007 a 31 de outubro de 2008, na Unidade de Pesquisa do Instituto Federal do Norte de Minas Gerais (IFNMG), Campus Januária, que fica localizado na latitude $15^{\circ} 28^{\prime} 55^{\prime}$ ' S e longitude $44^{\circ} 22^{\prime} 41^{\prime}$ ', W, altitude $474 \mathrm{~m}$. O clima regional é classificado como Aw (tropical úmido com inverno seco e verão chuvoso) de acordo com Köppen. A precipitação média anual é de $850 \mathrm{~mm}$, a umidade relativa média é de $60 \%$ e a temperatura média anual é de $27^{\circ} \mathrm{C}$ (Jacomine et al., 1979).

Diariamente, às 09:00 $\mathrm{h}$, foram coletados os dados de temperatura do ar, da radiação solar, da umidade relativa do ar, da velocidade do vento a $2 \mathrm{~m}$ de altura e da precipitação pluvial de uma estação meteorológica automática. Com os dados climáticos, calculou-se a evapotranspiração de referência pelos seguintes métodos:

Método de Penman-Monteith (ET $T_{0}$ PM)

$$
E T 0=\frac{0,408 \Delta(R n-G)+\gamma \frac{900}{T+273} U_{2}\left(e_{s}-e_{a}\right)}{\Delta+\gamma\left(1+0,34 U_{2}\right)}
$$

em que:

$\mathrm{ET}_{0}=$ evapotranspiração de referência, em $\mathrm{mm} \mathrm{d}^{-1}$;

$\mathrm{Rn}=$ saldo de radiação à superfície, em $\mathrm{MJ} \mathrm{m}^{-2} \mathrm{~d}^{-1}$;

$\mathrm{G}=$ fluxo de calor no solo, em $\mathrm{MJ} \mathrm{m}^{-2} \mathrm{~d}^{-1}$;

$\mathrm{T}=$ temperatura do ar a $2 \mathrm{~m}$ de altura, em ${ }^{\circ} \mathrm{C}$;

$\mathrm{U}_{2}=$ velocidade do vento à altura de $2 \mathrm{~m}, \mathrm{em} \mathrm{m} \mathrm{s}^{-1}$;

$\mathrm{e}_{\mathrm{s}}=$ pressão de saturação de vapor, em $\mathrm{kPa}$;

$\mathrm{e}_{\mathrm{a}}=$ pressão de vapor atual do ar, em $\mathrm{kPa}$;

$\left(\mathrm{e}_{\mathrm{s}}-\mathrm{e}_{\mathrm{a}}\right)=$ déficit de pressão de vapor, em em $\mathrm{kPa}$;

$\square=$ declividade da curva de pressão de vapor de saturação, em $\mathrm{kPa}^{\circ} \mathrm{C}^{-1}$; e

$=$ constante psicrométrica, em $\mathrm{kPa}^{\circ} \mathrm{C}^{-1}$.

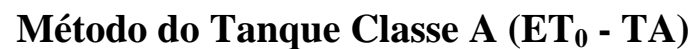

As medidas de evaporação da água do Tanque Classe A (EVCA) foram tomadas diariamente e os seus valores convertidos em lâminas evapotranspiradas pela Equação 2.

$$
E T 0=K t \quad x E V C A
$$

em que:

$\mathrm{ET}_{0}=$ evapotranspiração de referência, em $\mathrm{mm} \mathrm{d}^{-1}$;

EVCA = evaporação do tanque, em $\mathrm{mm} \mathrm{d}^{-1} ; \mathrm{e}$

$\mathrm{K}_{\mathrm{t}}=$ coeficiente do tanque.

Os valores do coeficiente do tanque para as condições locais foram obtidos utilizando-se a equação 3: 
TANQUE EVAPORIMÉTRICO ALTERNATIVO E EQUAÇÕES PARA ESTIMATIVA DA EVAPOTRANSPIRAÇÃO DE REFERÊNCIA NA REGIÃO NORTE DE MG

$$
\begin{gathered}
\mathrm{K}_{\mathrm{t}}=0.61+3,41 \times 10^{-3} \mathrm{UR}_{\text {med }}-1,62 \times 10^{-3} \mathrm{U}_{2} \mathrm{UR}_{\text {med }}-9,59 \times 10^{-6} \mathrm{U}_{2} \mathrm{R}_{\text {solo }}+3,27 \times 10^{-3} \mathrm{U}_{2} \ln \left(\mathrm{R}_{\text {solo }}\right)- \\
2,89 \times 10^{-3} \mathrm{U}_{2} \ln \left(86.4 \mathrm{U}_{2}\right)-10,6 \times 10^{-3} \ln \left(86.4 \mathrm{U}_{2}\right) \ln \left(\mathrm{R}_{\text {solo }}\right)+0,63 \times 10^{-3}\left[\ln \left(\mathrm{R}_{\text {solo }}\right)\right]^{2} \ln \left(86.4 \mathrm{U}_{2}\right)(3)
\end{gathered}
$$

em que:

$\mathrm{U}_{2}=$ velocidade média diária do vento a $2 \mathrm{~m}$ de altura $\left(\mathrm{m} \mathrm{s}^{-1}\right)$;

$\mathrm{UR}_{\text {med }}=$ umidade relativa média (\%); e

$\mathrm{R}_{\text {solo }}=$ menor distância do centro do tanque ao limite da bordadura solo nu (m).

\section{Método de Linacre $\left(\mathrm{ET}_{\mathbf{0}}-\mathrm{L}\right)$}

A evapotranspiração de referência pelo método de Linacre, em $\mathrm{mm} \mathrm{d}^{-1}$, pode ser obtida em função da altitude, latitude e das

$$
\text { ET0 }=\frac{700 \mathrm{~T}_{\underline{m}}}{2} \frac{(100-\alpha)+15\left(\mathrm{~T}_{\underline{a}}-\mathrm{T}_{\underline{d}}\right)}{\left(80-\mathrm{T}_{\mathrm{a}}\right)}
$$

em que:

$\mathrm{ET}_{0}=$ evapotranspiração de referência, em $\mathrm{mm} \mathrm{d}^{-1}$;

$\mathrm{T}_{\mathrm{m}}=\mathrm{Ta}+0,006 \mathrm{z}$

$\mathrm{z}$ - altitude, $\mathrm{m}$;

$\mathrm{T}_{\mathrm{a}}$ - temperatura média do ar, ${ }^{\circ} \mathrm{C}$;

$\alpha$-é a latitude local (graus); e

$\mathrm{T}_{\mathrm{d}}$ - é a temperatura de ponto de orvalho, ${ }^{\circ} \mathrm{C}$.

\section{Método de Hargreaves-Samani (ET $\left.\mathbf{T}_{\mathbf{0}}-\mathrm{H} \& \mathrm{~S}\right)$}

O método de Hargreaves \& Samani (1985) foi utilizado, conforme a Equação 5.

$\mathrm{ET} 0=\mathrm{aR}_{\mathrm{a}} \mathrm{TD}^{1 / 2}\left(\mathrm{~T}_{\mathrm{a}}+17,8\right)$

em que:

$\mathrm{ET}_{0}=$ evapotranspiração de referência, em $\mathrm{mm} \mathrm{d}^{-1}$;

$\mathrm{TD}=$ variação de temperatura $\left({ }^{\circ} \mathrm{C}\right)$ no dia;

$\mathrm{a}=$ coeficiente adimensional;

$\mathrm{R}_{\mathrm{a}}=$ radiação solar no topo da atmosfera $\left(\mathrm{mm} \mathrm{d}^{-1}\right)$; e

$\mathrm{T}_{\mathrm{a}}=$ temperatura média diária do $\operatorname{ar}\left({ }^{\circ} \mathrm{C}\right)$.

\section{Método do Minitanque (EV - MT)}

$\mathrm{O}$ tanque simplificado alternativo (Minitanque) foi confeccionado a partir de um tambor metálico com volume de $200 \mathrm{~L}$ e diâmetro interno de $57 \mathrm{~cm}$. O tambor foi cortado a uma altura de $45 \mathrm{~cm}$ da base. Em seguida, fez-se um orifício com diâmetro de $11,5 \mathrm{~mm}$, a $5 \mathrm{~cm}$ da borda superior, acoplando-se a este orifício um tubo metálico de $5 \mathrm{~cm}$ de comprimento. Este orifício serviu como referência para a medida da quantidade de água evaporada. Após a construção do tanque, ele foi pintado temperaturas diárias máxima, mínima e do ponto de orvalho, pela equação (Linacre, 1977): 


\section{TANQUE EVAPORIMÉTRICO ALTERNATIVO E EQUAÇÕES PARA ESTIMATIVA DA EVAPOTRANSPIRAÇÃO DE REFERÊNCIA NA REGIÃO NORTE DE MG}

\section{Análise dos dados}

Realizou-se a análise de regressão entre a evapotranspiração de referência estimada por Penman-Monteith (ET $\mathrm{ET}_{0}$ - PM) e aquelas obtidas pelo Tanque Classe A ( $\mathrm{ET}_{0}$ - TA), Linacre $\left(\mathrm{ET}_{0}-\mathrm{L}\right)$, Hargreaves \& Samani $\left(\mathrm{ET}_{0}-\mathrm{H} \& \mathrm{~S}\right)$ e a evaporação do Minitanque (EV - MT). A $\mathrm{ET}_{0}$ estimada por PenmanMonteith foi considerada variável independente e as demais, $\mathrm{ET}_{0}$ e a EV-MT, dependentes.

Consideraram-se três meios de estabelecer a referida análise: 1 - utilização do banco de dados de todo o período avaliado; 2 utilização do período abrangido de 22 de novembro de 2007 até 28 de abril de 2008 (época chuvosa); e 3 - utilização do período de 29 de abril de 2008 até 21 de novembro de 2007 (época seca).

As regressões foram realizadas utilizando-se o software estatístico SAEG, Versão 9.1 (SAEG, 2007).

\section{RESULTADOS E DISCUSSÃO}

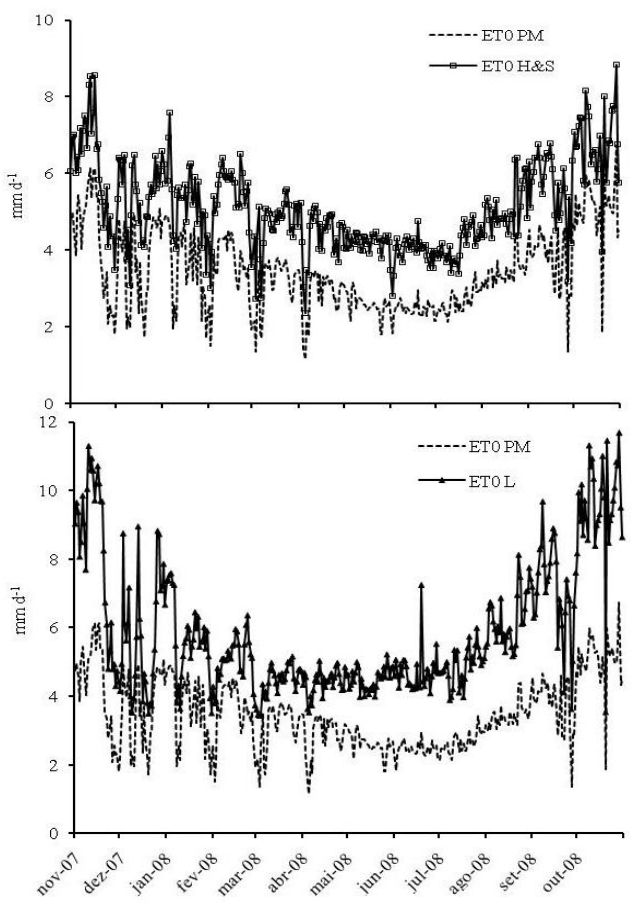

A $\mathrm{ET}_{0}$ obtida pelos métodos de HargreavesSamani, Tanque Classe A, Linacre e evaporação do minitanque superestimaram a $\mathrm{ET}_{0}$ obtida pelo método de PenmanMonteith em todo o período de condução deste trabalho (Figura 1). Em estudo com a cultura da melancia em Fortaleza - CE, o método de Hargreaves-Samani também superestimou a $\mathrm{ET}_{0}$ obtida pelo método de Penman-Monteith (Bezerra e Oliveira, 1999). Tagliaferre (2006) e Reis (2007) também concluíram que as estimativas de $\mathrm{ET}_{0}$ obtidas pelo método de HargreavesSamani superestimaram as obtidas pelo método de Penman-Monteith.

No estudo conduzido por Henrique e Dantas (2007) na cidade de Campina Grande- PB, o método de Linacre subestimou os valores de $\mathrm{ET}_{0}$ em relação ao método padrão, em todas as estações do ano. Diferenças entre os elementos climatológicos de Campina Grande - PB e de Januária - MG, levaram a subestimativa para a primeira cidade e a superestimava para a segunda de $\mathrm{ET}_{0}-\mathrm{L}$, quando comparado ao método padrão de estimativa da evapotranspiração

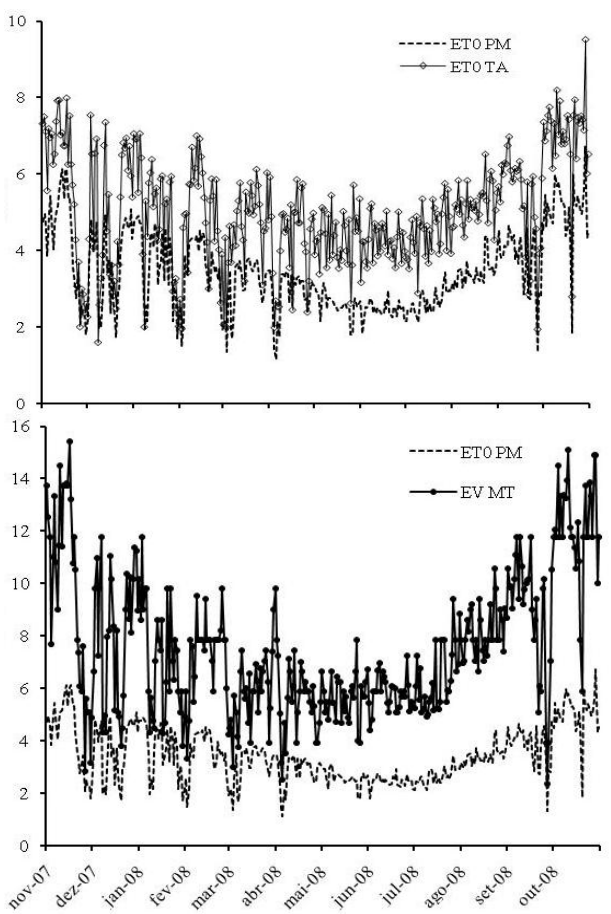

Figura 1. Evapotranspiração de referência obtida pelos métodos de Penman-Monteith $\left(\mathrm{ET}_{0}-\mathrm{PM}\right)$, Hargreaves e Samani $\left(\mathrm{ET}_{0}-\mathrm{H} \& \mathrm{~S}\right)$, Linacre $\left(\mathrm{ET}_{0}-\mathrm{L}\right)$, Tanque Classe A (ET $\left.{ }_{0}-\mathrm{TA}\right)$ e evaporação do Minitanque (EV-MT) para o período anual em Januária - MG. IFNMG - Campus Januária, Januária - MG. 

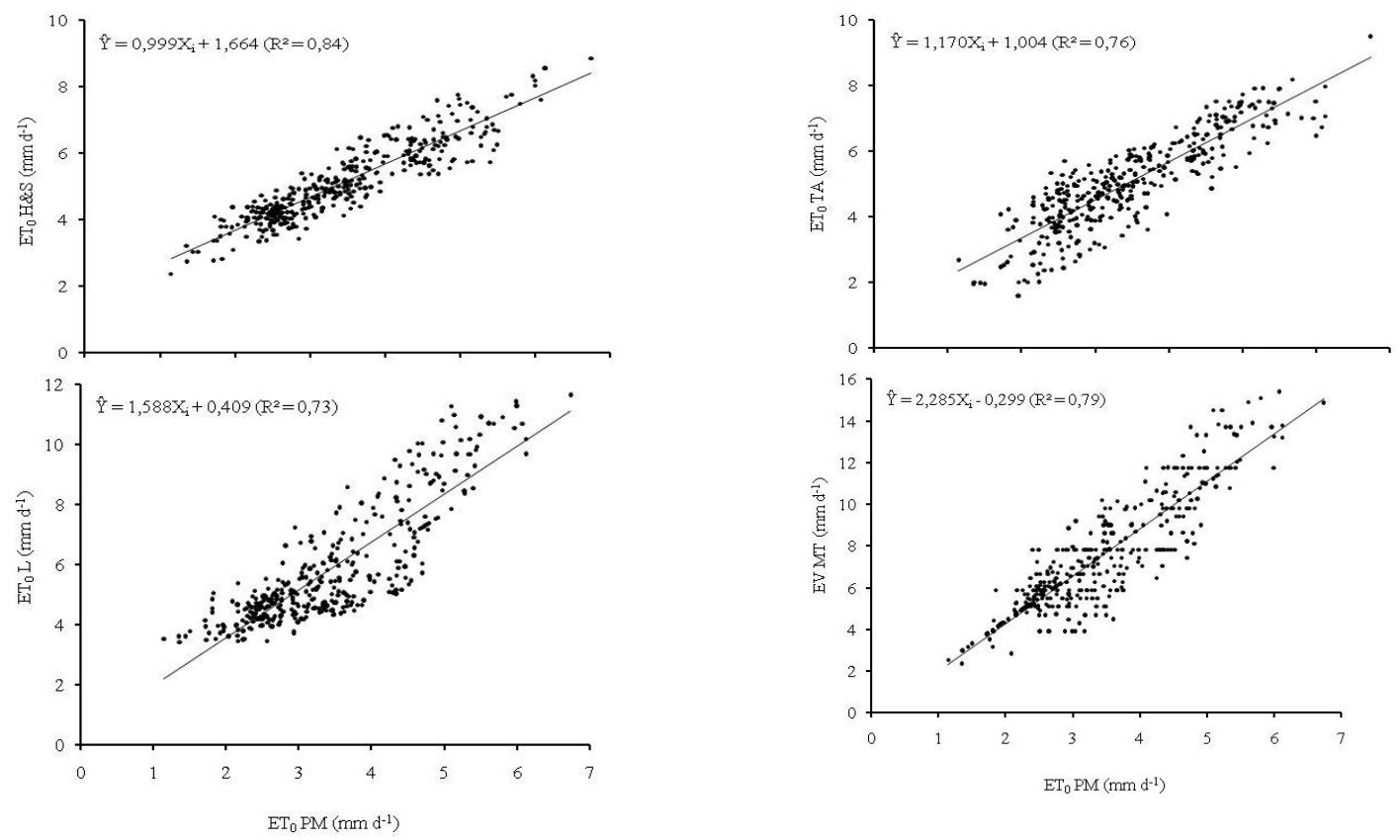

Figura 2. Regressões entre a evapotranspiração de referência obtida pelo método de PenmanMonteith $\left(\mathrm{ET}_{0}-\mathrm{PM}\right)$ e as obtidas por Hargreaves e Samani $\left(\mathrm{ET}_{0}-\mathrm{H} \& \mathrm{~S}\right)$, Linacre $\left(\mathrm{ET}_{0}-\mathrm{L}\right)$, Tanque Classe A (ET $\left.{ }_{0}-\mathrm{TA}\right)$ e a evaporação do Minitanque (EV-MT) para o período anual em Januária MG. IFNMG - Campus Januária, Januária - MG.

Os valores diários de EV obtidos pelo minitanque seguiram as variações observadas nos demais métodos avaliados para estimativa da $\mathrm{ET}_{0}$ (Figura 1). A estimativa da $\mathrm{EV}$ obtida pelo minitanque superou a $\mathrm{ET}_{0}$ obtida pelos métodos utilizados no trabalho para a obtenção de $\mathrm{ET}_{0}$. Isso se deve ao efeito de borda ou "Efeito Oásis", em que o ar quente e seco que constantemente chega à superfície do tanque com água livre aumenta sua evaporação. No tanque classe $\mathrm{A}$, tal efeito é corrigido pelo uso do coeficiente de tanque $\left(\mathrm{k}_{\mathrm{t}}\right)$, que ajusta a evaporação medida do tanque à sua $\mathrm{ET}_{0}$ (Allen et al., 2006).

Houve boa correlação entre os valores anuais de $\mathrm{ET}_{0}$ obtidos pelo método de PenmanMonteith e os estimados pelos métodos de Hargreaves-Samani, Linacre, Tanque classe A e a evaporação medida no minitanque (Figura 2).
Entre os métodos analisados, o que mostrou melhor correlação com o Penman-Monteith foi o de Hargreaves-Samani. Resultado semelhante foi constatado por Terra et al. (2008), por Gonçalves et al. (2009) e por Oliveira et al. (2010) para as cidades de Pelotas - RS, de Sobral - CE e de Juazeiro BA, respectivamente. No trabalho realizado por Reis et al. (2007) na cidade de Venda Nova dos Imigrantes - ES, com nove métodos para estimar a $\mathrm{ET}_{0}$, a menor correlação encontrada em escala diária foi com o método de Hargraves-Samani.

Houve superestimativa dos valores de $\mathrm{ET}_{0}$ obtidos pelos métodos estudados e da evaporação medida no minitanque em relação à $\mathrm{ET}_{0}$ obtida pelo método padrão FAO. A maior diferença das estimativas ocorreu entre a $\mathrm{ET}_{0}$ e a evaporação do minitanque (Figuras 1, 2, 3 e 4). 
TANQUE EVAPORIMÉTRICO ALTERNATIVO E EQUAÇÕES PARA ESTIMATIVA DA EVAPOTRANSPIRAÇÃO DE REFERÊNCIA NA REGIÃO NORTE DE MG
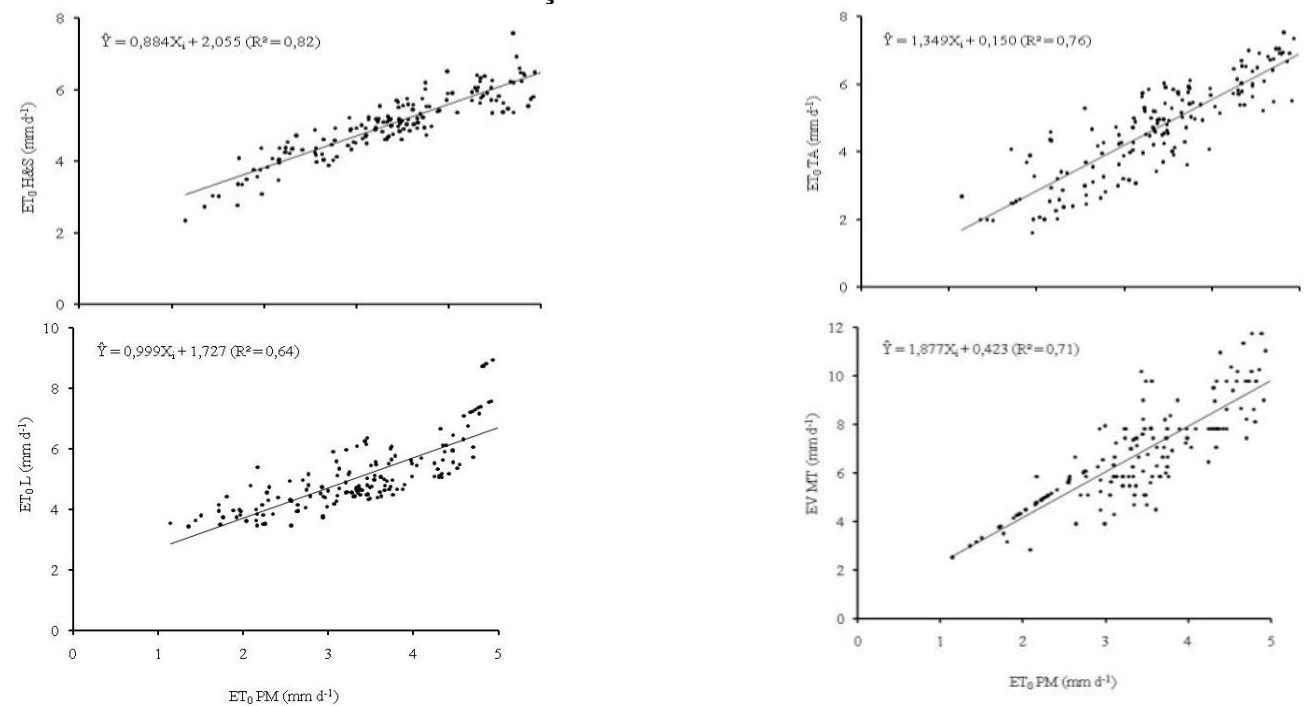

Figura 3. Regressões entre a evapotranspiração de referência obtida pelo método de PenmanMonteith $\left(\mathrm{ET}_{0}-\mathrm{PM}\right)$ e as obtidas por Hargreaves e Samani $\left(\mathrm{ET}_{0}-\mathrm{H} \& \mathrm{~S}\right)$, Linacre $\left(\mathrm{ET}_{0}-\mathrm{L}\right)$, Tanque Classe A (ET E $^{-\mathrm{TA}}$ ) e a evaporação do Minitanque (EV-MT) para o período com chuvas em Januária - MG. IFNMG - Campus Januária, Januária - MG.

Os maiores valores de evaporação medidos no minitanque podem ser justificados pela sua menor área, que se torna menos representativa das condições climáticas reinantes no local. Ainda, pequena espessura da chapa do tambor fornece maior energia à água, o que aumenta a evaporação. Além desses fatores, a proximidade do minitanque com o solo aumenta o fluxo de energia refletida recebida. Este é outro fator que concorre para maior evaporação medida

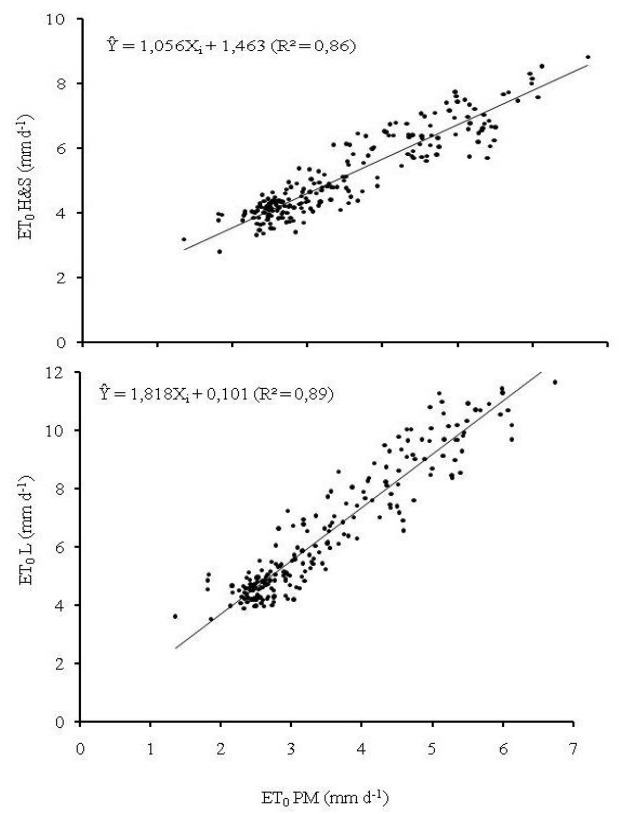

neste tanque, quando comparado, por exemplo, ao Tanque Classe A.

$\mathrm{O}$ desempenho dos métodos em estudo no período sem chuva foi melhor que no período com chuva (Figuras 3 e 4). No período com chuva, a diferença entre os valores de $\mathrm{ET}_{0}$ obtidos por Penman-Monteith e os demais métodos foi maior que no período sem chuvas. Ainda, os valores dos coeficientes de determinação das regressões entre a $\mathrm{ET}_{0}-\mathrm{PM}$ e os demais métodos foram menores no período chuvoso (Figura 3 ).

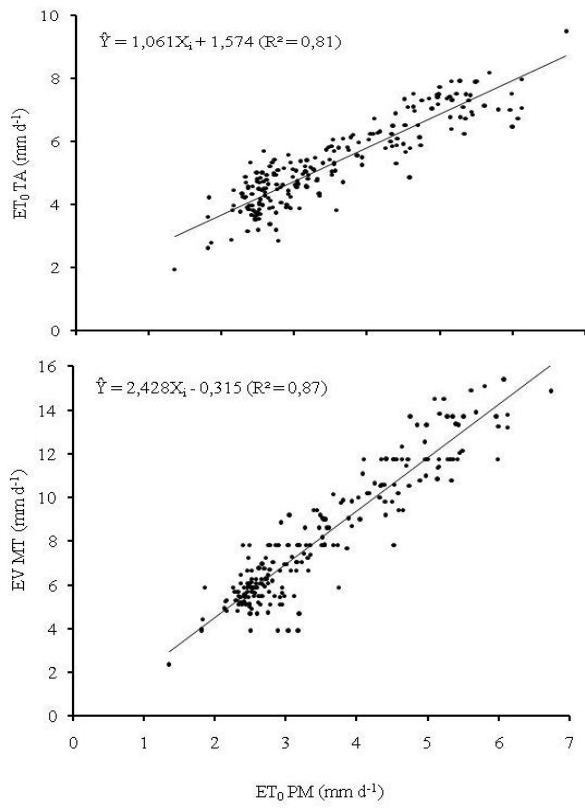

Figura 4. Regressões entre a evapotranspiração de referência obtida pelo método de Penman-Monteith $\left(\mathrm{ET}_{0}-\mathrm{PM}\right)$ e as obtidas por Hargreaves e Samani $\left(\mathrm{ET}_{0}-\mathrm{H} \& \mathrm{~S}\right)$, Linacre $\left(\mathrm{ET}_{0}-\mathrm{L}\right)$, Tanque Classe A $\left(\mathrm{ET}_{0}-\mathrm{TA}\right)$ e a evaporação do Minitanque (EV-MT) para o período sem chuvas em Januária - MG. IFNMG - Campus Januária, Januária - MG. 
A regressão da $\mathrm{ET}_{0}-\mathrm{PM}$ x $\mathrm{EV}-\mathrm{MT}$ apresentou maior coeficiente de determinação no período sem chuva em comparação à regressão $\mathrm{ET}_{0}-\mathrm{PM} \times \mathrm{ET}_{0}-$ Tanque Classe A. Entretanto, no período chuvoso, o maior coeficiente de determinação ocorreu na regressão $\mathrm{ET}_{0}-\mathrm{PM}$ $\mathrm{x} \mathrm{ET}_{0}-$ Tanque Classe A. Ambos os métodos medem a evaporação de uma superfície de água livre. A evaporação é muito influenciada pela umidade relativa do ar e vento. Tais elementos climáticos são mais variáveis no período chuvoso. A maior variabilidade desses elementos no período chuvoso, associada à menor área superficial do minitanque, leva a uma maior variabilidade dos valores de evaporação medidos neste último.

Henrique (2006), ao avaliar diferentes métodos para determinação da $\mathrm{ET}_{0} \mathrm{em}$ Campina Grande - PB, afirmou que nos dias em que há precipitação, tanto a temperatura como a irradiação solar diminuem. A precursora da evaporação da água em superfícies líquidas e dos solos úmidos é a irradiação. Como há diminuição na irradiação nos dias chuvosos, consequentemente, a $\mathrm{ET}_{0}$ é inferior à dos dias sem chuva.

A evaporação do minitanque apresentou boa correlação com os valores de $\mathrm{ET}_{0}$ estimados pelo método padrão FAO. No entanto, os valores da evaporação do minitanque superestimam os valores de $\mathrm{ET}_{0}-\mathrm{PM}$. Assim, é necessário um ajuste da evaporação do minitanque para a obtenção da $\mathrm{ET}_{0}$ conforme a mesma seja avaliada na estação com chuvas ou sem chuvas.

\section{CONCLUSÕES}

Os métodos de Hargreaves-Samani, Linacre e Tanque Classe A têm boa correlação na estimativa da $\mathrm{ET}_{0}$ com o método padrão FAO (Penman-Monteith);

Os métodos de estimativa da $\mathrm{ET}_{0}$ superestimaram a $\mathrm{ET}_{0}$ obtida pelo método de Penman-Monteith;
A evaporação medida no minitanque apresentou boa correlação e superestimou a $\mathrm{ET}_{0}$ estimada por Penman-Monteith;

Houve diferença na correlação das estimativas de $\mathrm{ET}_{0}$ dos diferentes métodos e da evaporação do minitanque com a $\mathrm{ET}_{0}$ estimada por Penman-Monteith nos períodos com e sem chuvas.

\section{AGRADECIMENTOS}

À Fapemig e ao CNPq, pela concessão de recursos financeiros para execução do trabalho e de bolsas de Iniciação Científica.

\section{REFERÊNCIAS BIBLIOGRÁFICAS}

ALLEN, R. G.; PEREIRA, L. S.; RAES, D.; SMITH, M. Evapotraspiración del cultivo. Utah State University. Guías para la determinación de los requerimientos de agua de los cultivos Paper, 56. Roma: FAO, 2006. $323 p$.

BERNARDO, S.; SOARES, A. A.; MANTOVANI, E. C. Manual de Irrigação. 8 ed. Viçosa. Editora UFV, 2006. 625p.

BEZERRA, F. M. L.; OLIVEIRA, C. H. C. Evapotranspiração máxima e coeficiente de cultura nos estádios fenológicos da melancia irrigada. Revista Brasileira de Engenharia Agrícola e Ambiental, Campina Grande, v. 3, n. 2, p. 173-177, 1999.

GONÇALVES, F. M.; FEITOSA, H. O.; CARVALHO, C. M.; GOMES FILHO, R. R.; VALNIR JÚNIOR, M. Comparação de métodos da estimativa da evapotranspiração de referência para o município de Sobral-CE. Revista Brasileira de Agricultura Irrigada, Fortaleza, v. 3, n. 2, p.71-77, 2009.

HARGREAVES, G. H.; SAMANI, Z. A. Reference crop evapotranspiration from teperature. Applied Engineering Agricuture, v.1, n.2, p.96-99, 1985. 
HENRIQUE, F. A. N. Estimativa da evapotranspiração de referência em Campina Grande-PB. 2006. 107 f. Dissertação (Mestrado em Climatologia Agrícola) - Universidade Federal de Campina Grande, Campina Grande.

HENRIQUE, F. A. N.; DANTAS R. T. Estimativa da evapotranspiração de referência em Campina Grande, Paraíba. Revista Brasileira de Engenharia Agrícola e Ambiental, Campina Grande, v. 11, n. 6, p. 594-599, 2007.

JACOMINE, P. K. T.; CAVALCANTI, A. C.; FORMIGA, R. A.; SILVA, F. B. R.; BURGOS, N.; MEDEIROS, L. A.; LOPES, O. P.; MELO FILHO, H. R. L.; PESSOA, S. G. P.; LIMA, P. C. Levantamento exploratório: reconhecimento de solos do Norte de Minas Gerais - área de atuação da Sudene. Recife: EMBRAPASNLCS/SUDENE-DRN, 1979. p. 10-11.

LINACRE, E. T. A simple formula for estimating evapotranspiration rates in various climates, using temperature data alone. Agricultural Meteorology, v.18, p.409-424, 1977.

OLIVEIRA, G. M.; LEITÃO, M. M. V. B. R.; BISPO, R. C.; SANTOS, I. M. S.; ALMEIDA, A. C. Comparação entre métodos de estimativa da evapotranspiração de referência na região Norte da Bahia. Revista Brasileira de Agricultura Irrigada, Fortaleza, v. 4, n. 2, p. 104-109, 2010.
REIS, E. F.; BRAGANÇA, R.; GARCIA, G. O.; PEZZOPANE, J. E. M.; TAGLIAFERRE C. Estudo comparativo da estimativa da evapotranspiração de referência para três localidades do estado do Espírito Santo no período seco. Idesia (Chile), v. 25, n. 3, p. 75-84, 2007.

SAEG - Sistema para Análises Estatísticas, Versão 9.1: Fundação Arthur Bernardes - UFV: Viçosa, 2007.

SILVA, V.P.R.; BELO FILHO, A.F.; SILVA, B.B.; CAMPOS, J.H.B.C. Desenvolvimento de um sistema de estimativa da evapotranspiração de referência. Revista Brasileira de Engenharia Agrícola e Ambiental, Campina Grande v.9, n.4, p. 547-553, 2005.

TAGLIAFERRE, C. Desempenho do irrigâmetro e de dois tipos de minievaporímetros para estimativa da evapotranspiração de referência. 2006. 110 f. Tese (Doutorado em Engenharia Agrícola) - Universidade Federal de Viçosa, Viçosa.

TERRA, V. S. S.; TEIXEIRA, C. F. A.; REISSER JÚNIOR, C.; MADALOZ, L. M.; STEINMETZ, S.; ALMEIDA, I. R.; TIMM, L. C. Evapotranspiração de referência estimada por diferentes métodos para a região de Pelotas- RS. In: CONGRESSO DE INICIAÇÃO CIENTIFÍCA， 18， 2008. Resumos... Pelotas: UFPel, p. 15- 20, 2008. 